\section{Somatic Depdc5 Deletion Recapitulates Electroclinical Features of Human Focal Cortical Dysplasia Type IIA}

\author{
Shuntong $\mathrm{Hu}, \mathrm{MD},{ }^{1,2}$ \\ Robert C. Knowlton, $\mathrm{MD}^{3}$ \\ Brendon O. Watson, $\mathrm{MD}, \mathrm{PhD}_{1}^{4}$ \\ Katarzyna M. Glanowska, $\mathrm{PhD}^{2,5}$ \\ Geoffrey G. Murphy, PhD, ${ }^{5}$ \\ Jack M. Parent, MD, 2,6 and \\ Yu Wang, MD, PhD ${ }^{2}$
}

Epileptogenic mechanisms in focal cortical dysplasia (FCD) remain elusive, as no animal models faithfully recapitulate FCD seizures, which have distinct electrographic features and a wide range of semiologies. Given that DEPDC5 plays significant roles in focal epilepsies with FCD, we used in utero electroporation with clustered regularly interspaced short palindromic repeats gene deletion to create focal somatic Depdc5 deletion in the rat embryonic brain. Animals developed spontaneous seizures with focal pathological and electroclinical features highly clinically relevant to FCD IIA, paving the way toward understanding its pathogenesis and developing mechanistic-based therapies.

ANN NEUROL 2018;84:140-146

$\mathrm{F}$ ocal cortical dysplasia (FCD) is a common cause of refractory focal epilepsies (FEs). As the most common underlying pathology in children with refractory FEs, it accounted for $26.8 \%$ of those cases in a series of approximately 2,600 specimens of brain tissue obtained during epilepsy surgery. ${ }^{1}$ FCD has been classified into several subtypes and entities on the basis of histopathology. In particular, FCD II is an isolated lesion characterized by disrupted cytoarchitecture including dysmorphic neurons without (FCD IIA) or with balloon cells (FCD IIB). ${ }^{1,2}$ Although FCD II is firmly linked to aberrant MTOR signaling pathways, ${ }^{3}$ a major challenge in understanding its epileptogenesis and intractability is to develop animal models that recapitulate its genetics, pathology, and electroclinical expression. For example, transgenic rodent, antimitotic agent, and irradiation models lack a focal lesion. Freeze lesions produce circumscribed lesions but fail to capture its genetics and pathologies. Recently, focal pathology was replicated in mouse models using in utero electroporation (IUE) to focally knockdown the mTOR inhibitor $T s c 1 / 2$ or overexpress mutant mTOR activators. ${ }^{4}$ However, a fundamental impediment to clinical translation is that none of these models had focal electrographic signatures of human FCD seizures such as paroxysmal fast activity (PFA) ${ }^{5}$ or fingerprints for the epileptogenic zone including low-voltage fast activity (LVFA). ${ }^{6}$ Whereas nearly all patients develop seizures with various semiologies, these FCD animals only displayed generalized tonic-clonic seizures (GTCs), and a significant number of them did not develop spontaneous seizures. ${ }^{7}$

The focal lesion seen in FCD II has led to the hypothesis that somatic mosaic mutations are the underlying genetic mechanism. Recent work has identified somatic activating mutations in $M T O R$ itself, genes encoding positive regulators of $M T O R$, and new genes as negative regulators of $M T O R$ via germline loss-of-function (LoF) mutation coupled to demonstrated or inferred somatic loss of the second allele. ${ }^{8}$ Germline or somatic DEPDC5 mutations are increasingly recognized as the common cause of familial or sporadic FEs including monogenic entities such as familial focal epilepsy with variable foci, autosomal dominant nocturnal frontal lobe epilepsy, and familial temporal lobe epilepsy. ${ }^{9}$ In addition to its role in magnetic resonance imaging (MRI)-negative FEs, DEPDC5 has also been associated with epileptogenic structural brain malformations, from FCD to large cortical malformations, such as hemimegalencephaly. ${ }^{8}$ DEPDC5 is part of a complex named GAP activity toward RAGs complex 1 (GATOR1), together with the proteins NPRL2 and NPRL3. As a key member of the amino acid sensing machinery, GATOR1 functions independently of TSC1/TSC2 signaling and directly inhibits mTORC1 recruitment to lysosomal membranes. ${ }^{10}$ However, no DEPDC5-related FEs animal model exists, and all Depdc5 transgenic animals reported to date failed to develop spontaneous seizures. All

From the ${ }^{1}$ Department of Neurology, Third Xiangya Hospital, Central South University, Changsha, China; ${ }^{2}$ Department of Neurology, University of Michigan, Ann Arbor, Ml; ${ }^{3}$ Department of Neurology, University of California, San Francisco, San Francisco, CA; ${ }^{4}$ Department of Psychiatry; ${ }^{5}$ Molecular and Behavioral Neuroscience Institute, University of Michigan, Ann Arbor, MI; and ${ }^{6}$ Ann Arbor VA Healthcare System, Ann Arbor, MI

Address correspondence to Dr Wang, Department of Neurology, 5025 BSRB, 109 Zina Pitcher Place, Ann Arbor, MI, 48109-2200. E-mail: eegwang@med. umich.edu

Additional supporting information can be found in the online version of this article.

Received Feb 21, 2018, and in revised form May 2, 2018. Accepted for publication Jun 10, 2018

View this article online at wileyonlinelibrary.com. DOI: 10.1002/ana.25272. 
homozygous knockout animals died embryonically, whereas heterozygous knockouts showed either no or subtle pathologies. ${ }^{11}$ Although a neuron-specific Depdc5 conditional knockout mouse survived to adulthood with severe neurological phenotypes, spontaneous focal electroclinical seizures were not recorded. ${ }^{11}$ Given its significant role in FEs and unique function in MTOR pathway, we performed IUE with clustered regularly interspaced short palindromic repeats (CRISPR) gene editing (CRISPR-IUE) on rat brains at embryonic day (E) 13 to E14 to introduce a focal region with somatic Depdc5 deletion and generated animals with pathological and electroclinical expression highly similar to human FCD IIA.

\section{Materials and Methods}

\section{Plasmids, IUE, and Animals}

Two Depdc5 guide RNAs (gRNAs) were designed using an online program (http://crispr.mit.edu) as previously described, and subcloned into the PX330 vector (plasmid 42230; Addgene, Cambridge, MA). ${ }^{12}$ Depdc5B was designed to target exon 12: 5'GGTGGTGCAGAACGAGAGAA $-3^{\prime}$, and $D e p d c 5 \mathrm{M}$ was designed to target exon 8: 5'- GTTCCGTTCTACGTCGGCTA-3'. The faithfulness scores were 95 and 98 , respectively. pCAG-green fluorescence protein (GFP), piggyBac donor plasmid (PBCAG-eGFP), and helper plasmid encoding piggyBac transposase (PBCAG-PBase) were obtained from Dr Joseph LoTurco (University of Connecticut). ${ }^{13}$ IUE was performed on rats (Sprague-Dawley; Charles River, Wilmington, MA) as previously described. ${ }^{12}$ The final
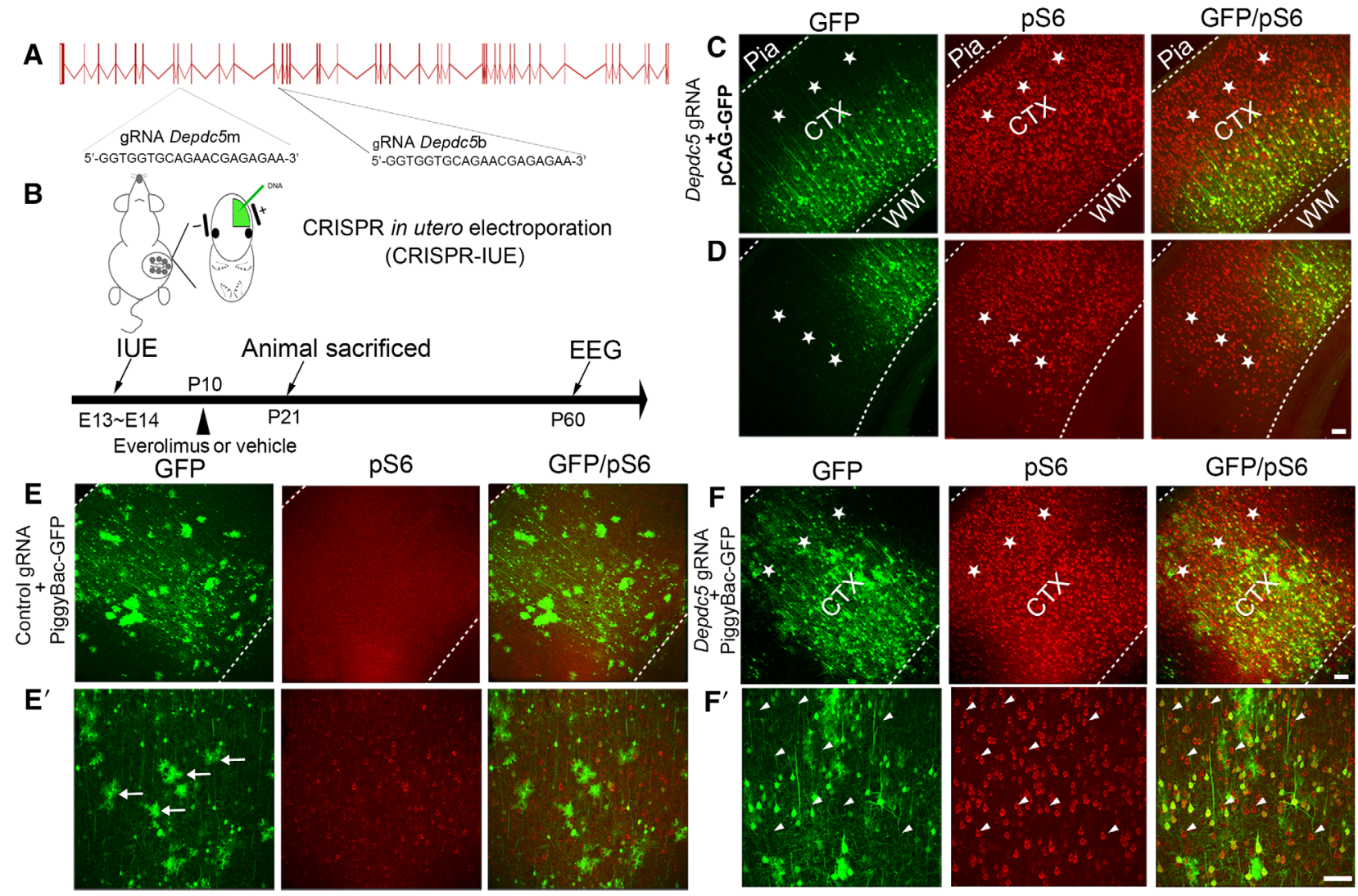

GFP/pS6

$\mathbf{E}^{\prime}$
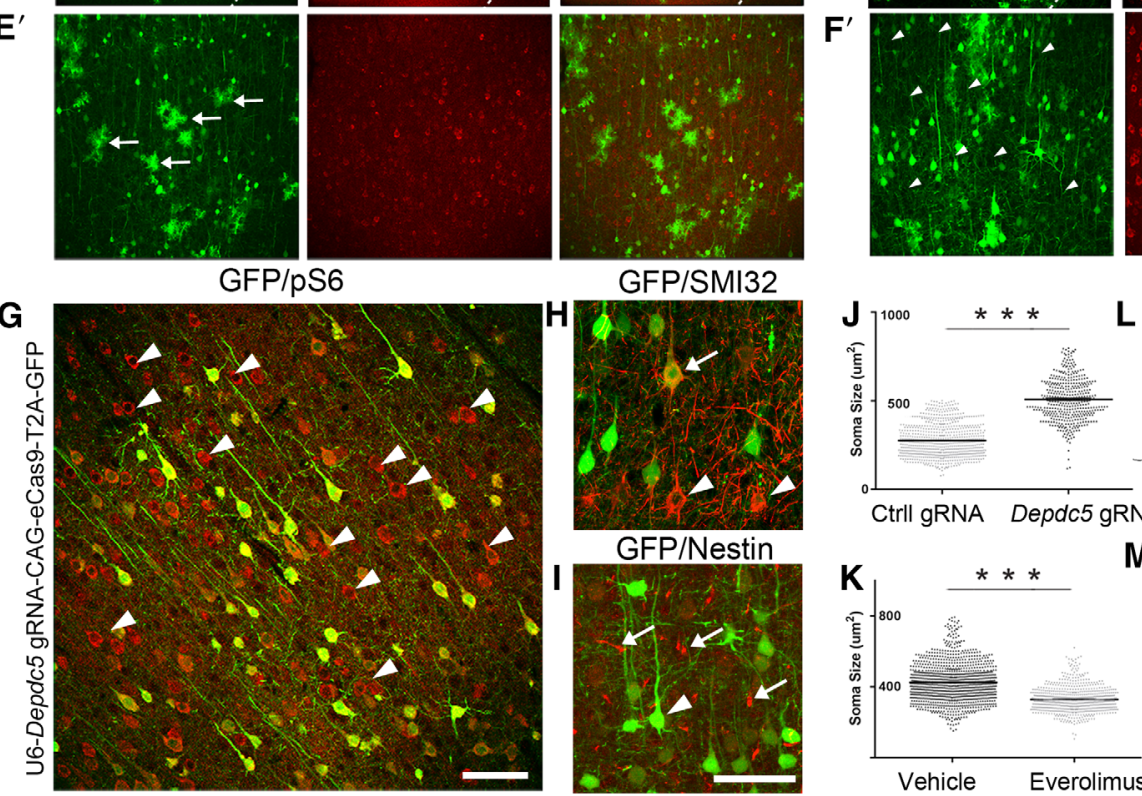

Ctrl I gRNA

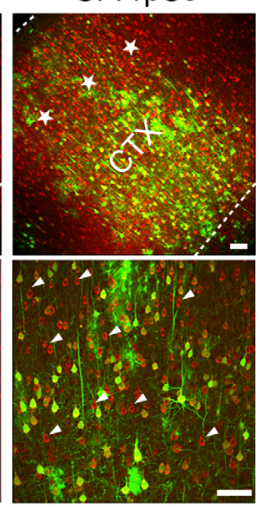

Depdc5 gRNA
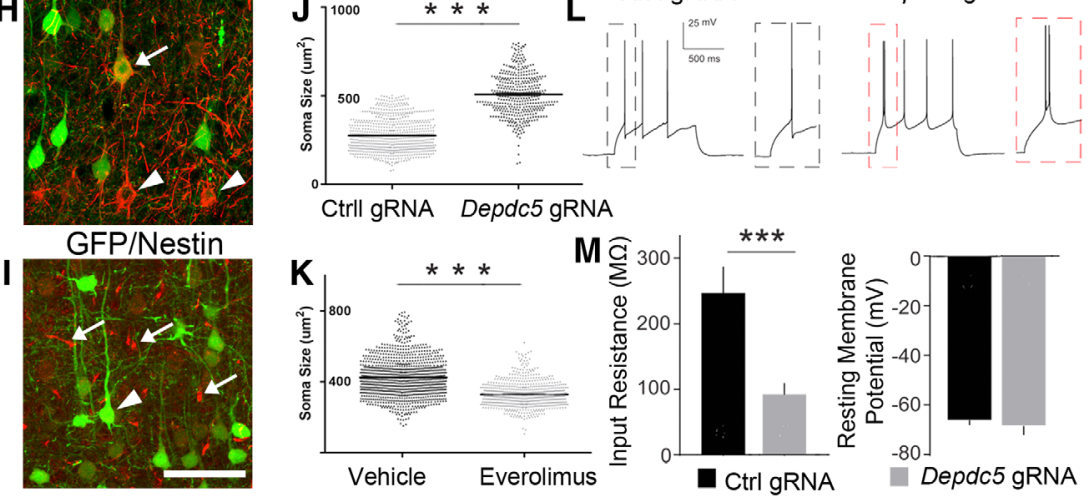

FIGURE 1: 
concentrations used for transfections were $0.5 \mu \mathrm{g} / \mu \mathrm{l}$ for eGFP and $1.5 \mu \mathrm{g} / \mu \mathrm{l}$ for Depdc5 CRISPR gRNA/Cas9. To track the entire lineage of transfected neural progenitors, PBCAG-eGFP $(0.5 \mu \mathrm{g} / \mu \mathrm{l})$ and pCAG-PBase $(1 \mu \mathrm{g} / \mu \mathrm{l})$ were coelectroporated with Depdc5 CRISPR gRNA/Cas9. pCAG-eCas9-T2A-GFP-U6-gRNA plasmid (a gift from Jizhong Zou; Addgene plasmid \# 79145) was used at $1.5 \mu \mathrm{g} /$ $\mu \mathrm{l}$. All animal studies were approved by the institutional animal care and use committee of the University of Michigan.

\section{Next Generation Sequencing}

To estimate the rate of somatic Depdc5 LoF mosaicism in the cortex, Depdc5 CRIPSR-IUE-transfected cortex was resected under the fluorescent dissection microscope. Targeted genomic areas flanking gRNA binding sites were polymerase chain reaction (PCR)-amplified using Platinum High Fidelity Taq DNA polymerase (11304-011; Invitrogen, Carlsbad, CA). PCR products were sent for deep sequencing to the Center for Computational and Integrative Biology, Massachusetts General Hospital, Harvard. Primers used for targeted sequencing were forward $5^{\prime}$ CCTTGAAACGTTGTAATGAACCCGA, and reverse primer 5'TGACACACAGATGCAGTACCT.

\section{Long-Term Electroencephalographic Recording and Analysis}

To determine if rats that underwent Depdc5 CRISPR-IUE exhibit electroclinical seizures and interictal epileptiform discharges (IEDs), we monitored animals with continuous videoelectroencephalography (EEG). Rats were implanted with 4 epidural screw electrodes at P60. Procedures for affixing electrodes were performed as previously described. ${ }^{14}$ Four burr holes were made, and electrodes were positioned and fastened (left and right parietal, 1 cerebellar, and 1 reference posteriorly over the sinus cavity) using mounting screws (E363/20; PlasticsOne, Roanoke,
VA). The sockets were fitted into a 6-pin electrode pedestal, and the entire apparatus was secured with dental cement (Stoelting, Wood Dale, IL). One week after surgery, animals were monitored continuously for 3 to 14 days by video-EEG recording (Natus, Middleton, WI). Recordings were sampled at $4,056 \mathrm{~Hz}$ and were analyzed offline with concurrent video. Seizures and epileptiform activity were assessed manually in their entirety by fellowship-trained and board-certified epileptologists blind to experiments. IEDs are described as transients distinguishable from background activity with a characteristic morphology, typically but neither exclusively nor invariably found in interictal EEGs of people with epilepsy. Epileptiform patterns have to fulfill at least 4 of the following 6 criteria: (1) di- or triphasic waves with sharp wave or spike morphology $(<200$-millisecond duration); (2) different wave duration than the ongoing background activity; (3) asymmetry of the waveform, a sharply rising ascending phase and a more slowly descending phase, or vice versa; (4) transient is followed by an associated after-going slow wave; (5) background activity surrounding epileptiform discharges is disrupted by the presence of the epileptiform discharges; and (6) distribution of the negative and positive potentials on the scalp suggests a source of the signal in the brain. ${ }^{15}$ Seizures are defined as EEG phenomenon consisting of repetitive epileptiform EEG discharges at $>2$ cycles/s and/or characteristic pattern with quasi-rhythmic spatiotemporal evolution (ie, gradual change in frequency, amplitude, morphology, and location), lasting at least several seconds (usually $>10$ seconds). Two other short duration ( $<10$ seconds) EEG seizure patterns are defined as electrodecrement or LVFA seen during clinically apparent epileptic seizures. EEG seizure patterns unaccompanied by behavioral epileptic manifestations are referred to as electrographic or subclinical seizures. ${ }^{15}$ For detection of sleep state, EEG voltage data were converted into a time-frequency power graph using a multitaper

FIGURE 1: Depdc5 clustered regularly interspaced short palindromic repeats (CRISPR)-in utero electroporation (IUE) leads to focal cortical mTOR hyperactivation. (A) Two short guide RNAs (gRNAs) are used to target rat Depdc5 either at exon 8 or exon 12. (B) CRISPR-IUE experiments are performed at embryonic day (E) 13 to E14. Animals are euthanized at postnatal day (P) 21 days for pathology evaluation or are treated with everolimus for 11 days beginning at P10. Video-electroencephalographic (EEG) monitoring starts at P60. (C, D) Coelectroporation of PCAG-green fluorescent protein (GFP) and Depdc5 CRISPR significantly increases pS6 immunoreactivity in both GFP-positive and GFP-negative cells. Stars indicate cell non-autonomous mTOR hyperactivation in the overlying (C) and adjacent cortices (D). To show that increased pS6 in GFP-negative cells is not due to dilution of episomal GFP transfection by dividing cells, Depdc5 CRISPR was coelectroporated with a piggyBac transposon system encoding GFP (see Materials and Methods) that integrates into the genome of neural progenitors. (E) In control CRISPR-transfected brains, baseline pS6 immunoreactivity is minimal. (F) In Depdc5 CRISPR-transfected brains, pS6 expression still markedly increases in both GFP-positive and GFP-negative cells. (E') Higher magnification images of E. GFP-labeled glial cells (arrows) suggest successful genome integration of GFP plasmid. (F') Higher magnification images of F. Many GFP-negative cells show strong pS6 immunoreactivity (arrowheads), suggesting cell nonautonomous mTOR hyperactivation. (G) Because plasmid cotransfection efficiency is not $100 \%, \mathrm{GFP}^{-} / \mathrm{pS}^{+}$neurons could be neurons transfected with only Depdc5 CRISPR plasmid. To ensure that all neurons undergoing Depdc5 CRISPR deletion are labeled with GFP, we electroporated a single vector plasmid that encodes eCAS9, Depdc5 gRNA, and GFP. With this approach, increased pS6 is still observed in GFP-negative cells (arrowheads), indicating cell non-autonomous mTOR hyperactivation. (H) An antibody to a nonphosphorylated neurofilament protein (SMI32) reveals aberrant cytoplasmic accumulation in both GFP-positive (arrows) and GFP-negative neurons (arrowheads). (I) Positive filamentous nestin staining (arrows), a typical pattern in reactive astrocytes, is seen in the dysplastic cortex. However, balloon cells, a pathological hallmark of focal cortical dysplasia IIB, which show patchy and accumulated nestin staining in the cell body, are never seen in Depdc5 CRISPR-transfected brains. Arrowhead indicates that a hypertrophic GFP-positive cell is negative for nestin staining. (J) The soma size of Depdc5 CRISPR-transfected neurons ( $N=3$ rats, $n=412$ cells) is nearly doubled versus control cells $(N=3, n=715)$. ***Two-tailed $t$ test, $p=0.002$. (K) Postnatal administration of everolimus partially rescues cytomegalic neurons. Drug group: $N=3, n=661$; no-drug vehicle group: $N=3, n=1,096$. ***Two-tailed $t$ test, $p=0.005$. (L, M) All cortical neurons with Depdc5 deletion fire initial doublet action potentials (none in control), suggesting increased intrinsic excitability (L), and show decreased input resistance (M). $N=3$ rats. $* * *$ Two-tail $t$ test, $p<0.05$. Scale bar $=100 \mu \mathrm{m}$. CTX $=$ cortex; WM $=$ white matter. 
method at 1-second resolution. Each second was automatically classified into non-rapid eye movement or wakelike states based on bimodality tests of cortical signals as previously described. ${ }^{16}$ Please see code in SleepScoreMaster.m file in the GitHub repository at https://github.com/buzsakilab/buzcode.

\section{Immunocytochemistry}

Brains were removed and fixed in $4 \%$ paraformaldehyde in phosphate-buffered saline after transcardial perfusion, sectioned at 50 to $80 \mu \mathrm{m}$ on a vibratome (VT1000S; Leica, Wetzlar, Germany), and processed for immunocytochemistry as free-floating sections. $^{12}$ Primary antibodies included mouse anti-pS6 (1:2,000, \#2211; Cell Signaling Technology, Danvers, MA), chicken anti-GFP (1: 1,000, GFP-1020; Aves Labs, Tigard, OR), mouse antinestin (1:20, clone rat-401; Millipore, Billerica, $\mathrm{MA}$, mouse anti-nonphosphorylated neurofilament $\mathrm{H}$ (1: 200, clone \#SMI32; BioLegend, San Diego, CA). Fluorescently conjugated secondary antibodies (Alexa Fluor 488, 594, or 647) were obtained from Molecular Probes (Eugene, OR), and nuclei were labeled with Bisbenzimide (H1398, Molecular Probes).

\section{Brain Slice Preparation, Electrophysiological Recordings, and Data Analysis}

Male and female rats (P21-P28) that previously underwent IUE at E13 were used for electrophysiological recordings. Slices were prepared as previously described. ${ }^{12}$ Electrophysiological recordings were made in a recording chamber mounted on the stage of an upright microscope (customized Scientifica [Uckfield, UK] optical platform). Slices were continuously perfused (miniplus 3; Gilson, Middleton, WI) with artificial cerebrospinal fluid containing $1 \mu \mathrm{M}$ tetrodotoxin, $20 \mu \mathrm{M}$ 6-cyano-7-nitroquinoxaline2,3-dione, and $20 \mu \mathrm{M} \mathrm{d}(-)$-amino-5-phosphonovaleric acid to block action potential firing and ionotropic glutamate currents at a rate of 5 to $6 \mathrm{ml} / \mathrm{min}$ at $31-32^{\circ} \mathrm{C}$ (in-line heater TC-324B; Warner Instruments, Hamden, CT). GFP-expressing cells in cortical layers 2 and 3 were targeted for recordings. Resistance micropipettes (3-5M $\Omega$ ) were filled with an internal solution containing (in mM) 100 Cs-gluconate, 0.2 ethyleneglycoltetraacetic acid, $5 \mathrm{MgCl}_{2}, 40$ hydroxyethylpiperazine ethane sulfonic acid, 2 Mg-ATP, 0.3 Na-GTP, 1 QX-314, pH 7.2. Data were acquired at $20 \mathrm{kHz}$ and filtered at $5 \mathrm{kHz}$. Only cells with stable passive properties were further analyzed. We collected data from a maximum of 3 neurons per animal to avoid bias. Statistical analysis was performed using Prism (GraphPad Software, La Jolla, CA). Average values for each of the variables were calculated per cell and further averaged for each experimental group. Parametric and nonparametric statistical analyses were performed as dictated by data distribution.

\section{Image Acquisition, Soma Size Measurements, and Statistical Analyses}

Multichannel imaging was performed using a Leica SP5 confocal microscope. All the images were further processed with Photoshop CS3 software (Adobe Systems, San Jose, CA). The soma size of GFP-positive neurons from P21 P30 brains were compared between control and Depdc5 CRISPR IUE animals. Automated measurement was performed in ImageJ. Statistical analysis was performed using Excel (Microsoft, Redmond, WA) and GraphPad. Three or more biological replicates were used for all studies. A confidence interval of $95 \%(p<0.05)$ was required for values to be considered statistically significant. All data are presented as mean \pm standard error of the mean unless noted otherwise.

\section{Results}

Phosphorylated ribosomal protein S6 (pS6) is commonly used as a marker of $\mathrm{mTORC} 1$ activity because $\mathrm{mTORC} 1$ activates p70 S6 kinase 1, which phosphorylates S6. ${ }^{10}$ To examine mTORC1 hyperactivation, immunostaining for pS6 was performed on brain sections from 3-week-old rats that were coelectroporated with a Depdc5 gRNA/Cas9 construct and a GFP-encoding plasmid (Fig 1). After Depdc5 CRISPR-IUE, pS6 immunoreactivity and soma size significantly increased in GFP-expressing neurons as compared to control CRISPR transfected brains. Interestingly, cell non-autonomous mTOR hyperactivation was observed in GFP-negative neurons with substantial extension to overlying and adjacent cortex, in line with clinical data indicating that histological and electrographic abnormalities occur beyond MRI-visualized margins. ${ }^{17}$ Antibodies to nonphosphorylated neurofilament proteins (SMI32) revealed aberrant cytoplasmic accumulation of neurofilament proteins in the dysplastic cortex of Depdc5 CRISPR-IUE rats, consistent with pathological findings observed in human FCD II. ${ }^{2}$ However, nestin-positive cells, a hallmark of balloon cells in human FCD IIB, were not observed. These findings suggest that focal Depdc5 somatic mutations in rat cortex generate human FCD IIA pathologies. Although we did not quantify the efficiency of Depdc5 gRNAs, we and other groups have shown that IUE-CRISPR achieves gene knockout efficiency of $>80 \% .^{12,18}$ The specificity of Depdc5 deletion was confirmed by finding similar cellular and electrographic phenotypes using gRNAs targeting 2 different Depdc5 exons. Specificity was further supported by pharmacological rescue experiments showing that treatment with the rapamycin analog everolimus normalized soma size. To estimate the degree of somatic Depdc5 LoF mosaicism, genomic DNA was extracted from the dysplastic cortex resected under fluorescent microscope. Targeted genomic areas flanking gRNA binding sites were PCR amplified and sent for next generation sequencing $(>70,000$ reads). The rate of Depdc5 LoF mosaicism ranged from $\sim 2.64 \%$ to $\sim 3.22$ $\%(\mathrm{n}=3)$. Whole cell patch clamp recordings of acute cortical slices showed firing of doublet spikes and decreased input resistance in GFP-labeled cortical neurons with Depdc5 deletion.

Critically, 100\% (11/11 from > 3 different litters) of rats undergoing Depdc5 IUE-CRISPR developed frequent focal IEDs and spontaneous seizures. IEDs were restricted to the hemisphere with dysplastic tissue, in contrast to 


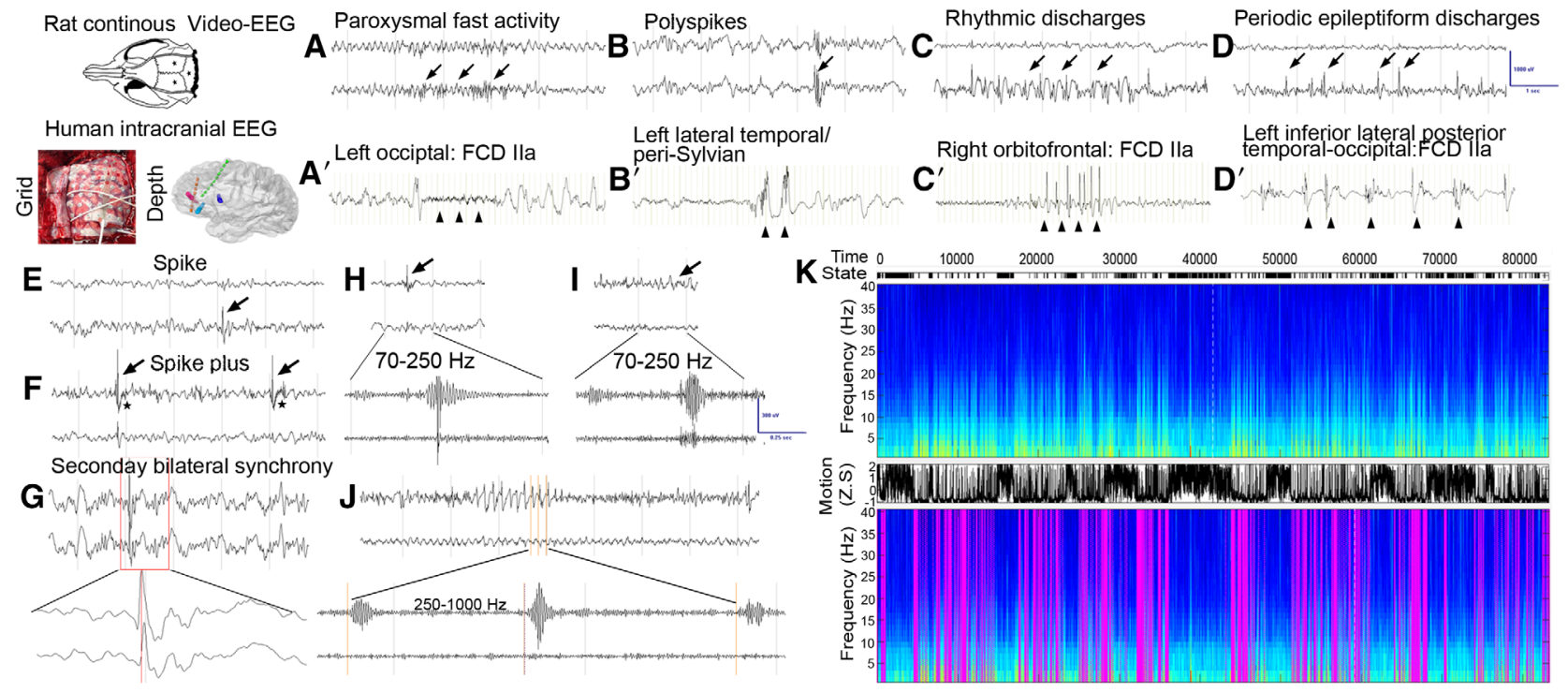

FIGURE 2: Interictal discharges highly resemble human focal cortical dysplasia (FCD). For all rat electroencephalographic (EEG) recordings, the upper trace is from an electrode implanted in the right parietal head region and the lower trace is from the left (see Materials and Methods). (A-D) Interictal EEG recordings from rats with Depdc5 CRISPR-IUE, including paroxysmal fast activity (A), polyspikes (B), rhythmic spike discharges (C), and periodic lateralized epileptiform discharges (D), are lateralized to the lesional hemisphere, and are highly similar to intracranial subdural or depth EEG recordings from patients with FCD Ila, including one with a DEPDC5 mutation (p.Tyr281Phe; $\left.A^{\prime}-D^{\prime}\right) .^{22}$ For all human intracranial recordings, shown is 1 electrode recording that captures the most representative EEG discharges. (E, F) Other typical focal interictal epileptiform discharge (IED) examples, including single focal spikes $(E)$ and spikes with overriding fast activity (stars) with minimal electrical field to the opposite hemisphere (F). (G) Rapid bilaterally synchronous-appearing IEDs are seen occasionally, but they display lateralized onset based upon asymmetric amplitude and lead-in time (red vertical line). (H) Ripples associated with a spike are displayed with a $70-$ to $250 \mathrm{~Hz}$ frequency band. (I) Ripples that are not associated with spikes. (J) Fast ripples associated with a train of spikeand-wave discharges are displayed with a 250 - to $1,000 \mathrm{~Hz}$ frequency band. These high-frequency oscillations are also lateralized to the lesional hemisphere. (K) Shown is a spectrogram of oscillatory power (color) versus time (horizontal) across various frequencies at each time point (vertical). IEDs, shown as purple lines in the lower power spectral image, occur predominantly in non-rapid eye movement sleep, recognized by increased lower frequency band power and lower motion scores. See upper panel for fully visible spectrogram and see Materials and Methods. To quantify IEDs during sleep versus wakefulness, IEDs were counted during 1 hour of sleep and wakefulness for 3 days from 3 rats with Depdc5 CRISPR-IUE. The average number of IEDs is $32.89 \pm 6.326$ during sleep and $7 \pm 3$ during wakefulness ( 2 -tailed $t$ test, $p=0.002$ ).

generalized discharges with current FCD models (Fig 2). Importantly, PFA, rhythmic spikes, and periodic lateralized epileptiform discharges (PEDs), hallmarks of human FCD II, ${ }^{5}$ were detected in the rats as well as our FCD II patients. High-frequency oscillations was also lateralized to the lesional side, supporting focal epileptogenicity. IEDs were also highly sleep-dependent. Ictally, electrographic seizure onsets were lateralized to the lesional hemisphere, and the common onset patterns in patients with FEs, including focal LVFA, rhythmic spikes, background suppression, and PEDs, ${ }^{6}$ were frequently observed (Fig 3A, C, D). These electrographic features were similar to those seen with intracranial ictal EEG recordings in FCD II patients and 1 patient with a pathological Depdc5 mutation (see Fig 3B). Semiologically, the clinical seizure expression in rats, including behavioral arrest, tonic stiffening, and contralateral clonic activity (Supplementary Videos 1-5), more closely recapitulates the variability seen in human FCD than existing models that show limited semiologies or only GTCs. ${ }^{7}$ For example, behavioral arrest was commonly observed in these rats, similar to human complex partial seizures. Many seizures presented with subtle clinical/ behavioral changes, such as waking up and subtle trunk tonic posturing. GTCs occasionally occurred late into the seizure but never occurred at the onset.

\section{Discussion}

To our knowledge, this is the first animal model that recapitulates genetics, pathology, and most importantly, electroclinical features of human FCD IIA. We did not observe nestin-positive balloon cells that are typically seen in human FCD IIB. ${ }^{3}$ Considering the striking difference in neurogenesis between humans and rodents, particularly the absence of outer radial glia cells in rodents, ${ }^{13}$ it is probably not a surprise that a rodent model lacks certain cytoarchitectural features. In addition, balloon cells have been shown to be silent electrographically. ${ }^{19}$ Genetically, germline DEPDC5 mutations occur more often than all other mutations in FE-related genes combined ${ }^{9}$ and directly inhibit MTOR 
A

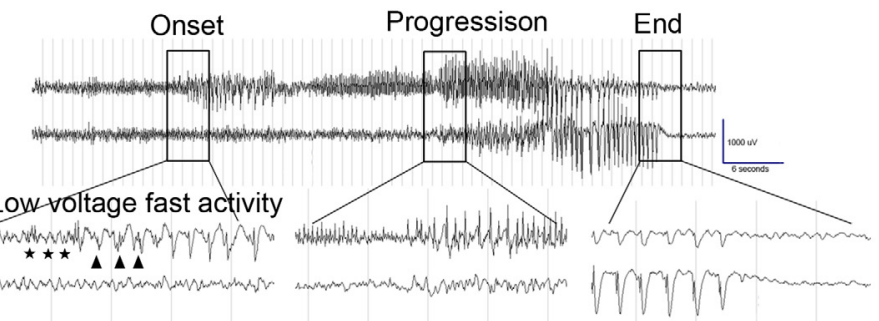

C Onset Progression End

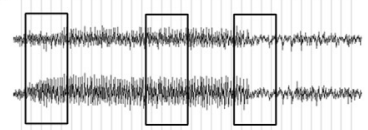

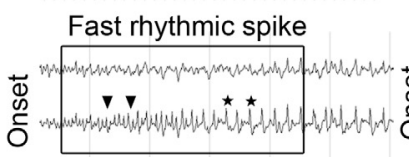

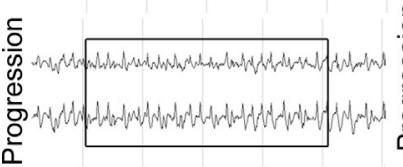

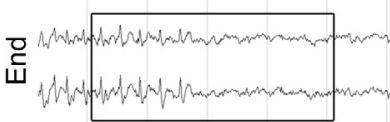

D Onset Progression End
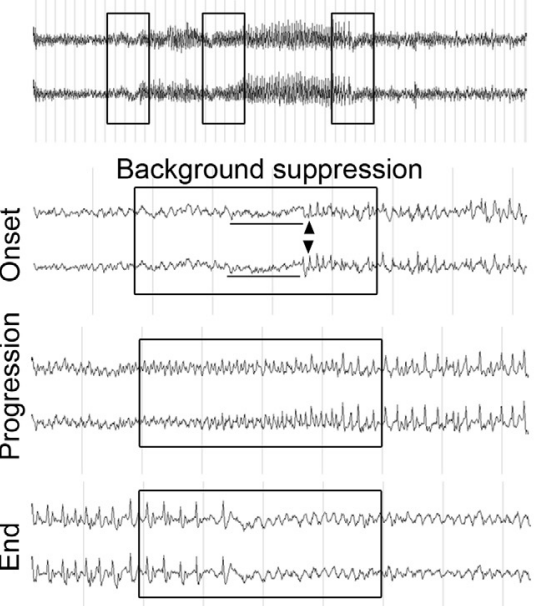

B

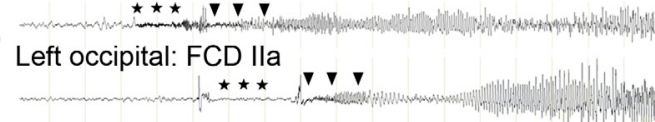

Left inferior lateral posterior temporal-occipital: FCD Ila $\star \star \star \star \nabla \nabla\rangle$

Right orbitofrontal: FCD Ila

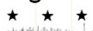

Left anterior insula / frontal operculum: FCD Ilb

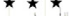

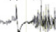

Left lateral temporal / peri-Sylvian: DEPDC5 mutation

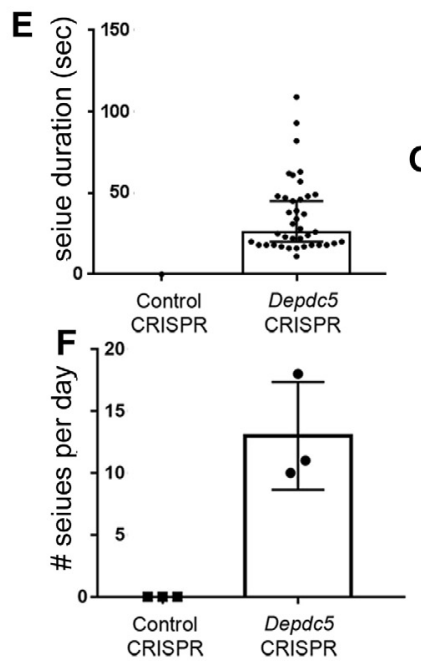

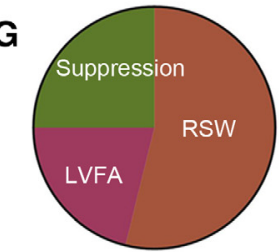

EEG onset patterns
H

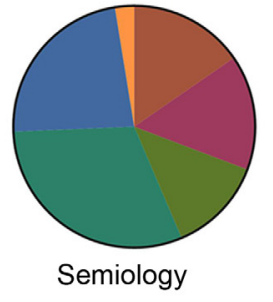

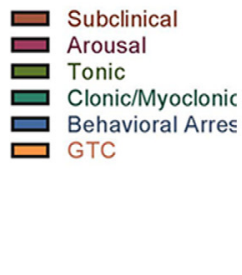
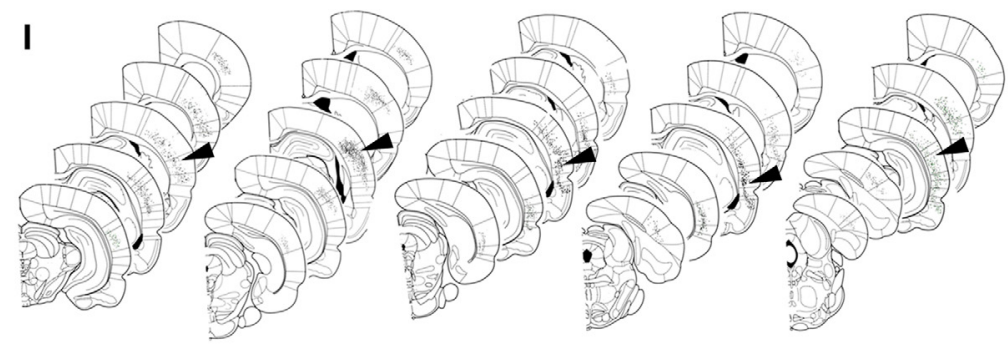

FIGURE 3: Electroclinical seizures highly resemble human focal cortical dysplasia (FCD). (A) Shown is an entire seizure from a rat with Depdc5 CRISPR-IUE. The top electroencephalographic (EEG) traces have a compressed time base of $5 \mathrm{~mm} / \mathrm{s}$, and the lower ones have a standard time base of $30 \mathrm{~mm} / \mathrm{s}$. The seizure starts with low-voltage fast activity (LVFA; stars) followed by $2-3 \mathrm{~Hz}$ spike and wave discharges (arrowheads). (B) Intracranial recordings from patients with FCD II show common ictal onset patterns including LVFA, background suppression, and bursts of rhythmic spikes. (C, D) Two additional representative seizures recorded from rats with Depec5 CRISRP-IUE show typical seizure onset patterns of rhythmic spikes (C) and background attenuation (D). (E-H) Thirtynine seizures from 3 different animals were analyzed for seizure duration $(E ; 35.97 \pm 22.85$ seconds, median $=26$ seconds), frequency (F; $13 \pm 2.5 / 24$ hours), EEG onset patterns (G), and semiology (H). The majority of seizures start with rhythmic spike discharges (RSW; 53.8\%), background suppression (25.0\%), and LVFA (21.2\%). Behaviorally, most seizures show clonus/myoclonus $(30.1 \%)$ or behavioral arrest (eg, freezing, stopping exploration; $23.1 \%)$. Other types include tonic posturing (12.8\%) and arousal without other clear clinical signs (15.1\%); $15.1 \%$ of electrographic seizures were subclinical. Notably, only 1 seizure progressed to a generalized tonic-clonic seizure (GTC). (I) Representative reconstructed brain sections show the size and position of Depdc5 CRISPR transfection (each black dot is a transfected neuron) at 5 rostrocaudal levels in 5 rats after EEG recording was completed at postnatal days 70 to 90 . Arrowheads indicate the section in each series with the most extensive Depdc5 CRISPR transfection.

independently of $\mathrm{TSC} 1 / \mathrm{TSC} 2 .^{10}$ Although DEPDC5 somatic mutations have been associated with brain malformations from FCD to hemimegalencephaly, ${ }^{9}$ how a germline mutation sustains a focal pattern in inherited epilepsies still remains enigmatic. A plausible emerging hypothesis is that DEPDC5 germline LoF mutation together with a second biallelic somatic hit in DEPDC5 itself leads to the development of FCD, known as "2-hit" mechanism, initially described in cancer and tuberous sclerosis. ${ }^{9}$ This biallelic inactivation hypothesis is also supported by a recent report of a DEPDC5 somatic nonsense variant in resected brain tissue from an individual with FCD IIA and a familial history of FEs due to a germline mutation in DEPDC5. ${ }^{9}$ Pathologically, both cell-autonomous and cell non-autonomous 
mTOR hyperactivation was observed in our model, in line with the clinical observation that FCD histologic and electrographic abnormality occurs beyond the MRI margin. ${ }^{17}$ Clinically, our Depdc5 CRISPR-IUE rats have a distinctly different electroclinical repertoire from current FCD models. The full spectrum of seizure semiologies and characteristic interictal and ictal discharges are unique and highly clinically relevant to human FCD. However, 2 important questions remain to be answered. First, cell non-autonomous mTOR hyperactivation, a surprising finding, likely plays an important role in epileptogenesis and overall clinical manifestations. However, the mechanism is completely unknown. Second, interneurons are firmly tied to ictogenesis and epileptogenesis in FCD. ${ }^{20}$ However, they are born in the ventral medial ganglionic eminence and lateral ganglionic eminence, ${ }^{21}$ which are not transfected by Depdc5 CRISPRIUE. A unifying hypothesis would be that cell nonautonomous mTOR hyperactivation impairs interneuron development. Our model should pave the way to study epileptogenesis and ictogenesis at cellular and circuit levels, as well as to develop mechanistic-based therapies.

\section{Acknowledgment}

Y.W. received funding from the NIH National Institute of Neurological Disorders and Stroke (NINDS; K08NS099379), Pediatric Epilepsy Research Foundation, and Citizens United for Research in Epilepsy. J.M.P. received funding from the NIH NINDS (R01NS058585). G.G.M. received funding from the NIH National Institute on Aging (R01AG052934). K.M.G. received funding from the American Epilepsy Society. B.O.W. received funding from the NIH National Institute of Mental Health (K08MH107662). R.C.K received funding from the NIH NINDS (U01NS09871). S.H. received funding from the China Scholarship Council (201606370207).

\section{Author Contributions}

Y.W. contributed to the conception and design of this study. All authors contributed to the acquisition and analysis of data. Y.W., J.M.P., and B.O.W. contributed to drafting the text.

\section{Potential Conflicts of Interest}

Nothing to report.

\section{References}

1. Blumcke I, Spreafico R, Haaker G, et al. Histopathological findings in brain tissue obtained during epilepsy surgery. N Engl J Med 2017;377: $1648-1656$
2. Blümcke I, Thom M, Aronica E, et al. The clinicopathologic spectrum of focal cortical dysplasias: a consensus classification proposed by an ad hoc task force of the ILAE Diagnostic Methods Commission. Epilepsia 2011;52:158-174

3. Iffland PH 2nd, Crino PB. Focal cortical dysplasia: gene mutations, cell signaling, and therapeutic implications. Annu Rev Pathol 2017; 12:547-571.

4. Becker AJ, Beck H. New developments in understanding focal cortical malformations. Curr Opin Neurol 2018;31:151-155.

5. Quirk JA, Kendall B, Kingsley DP, et al. EEG features of cortical dysplasia in children. Neuropediatrics 1993;24:193-199.

6. Grinenko O, Li J, Mosher JC, et al. A fingerprint of the epileptogenic zone in human epilepsies. Brain 2018;141:117-131.

7. Lim JS, Gopalappa R, Kim SH, et al. Somatic mutations in TSC1 and TSC2 cause focal cortical dysplasia. Am J Hum Genet 2017;100: $454-472$

8. D'Gama AM, Woodworth MB, Hossain AA, et al. Somatic mutations activating the mTOR pathway in dorsal telencephalic progenitors cause a continuum of cortical dysplasias. Cell Rep 2017;21:3754-3766.

9. Marsan E, Baulac S. Review: Mechanistic target of rapamycin (mTOR) pathway, focal cortical dysplasia and epilepsy. Neuropathol Appl Neurobiol 2018:44:6-17

10. Bar-Peled L, Chantranupong $L$, Cherniack $A D$, et al. $A$ tumor suppressor complex with GAP activity for the Rag GTPases that signal amino acid sufficiency to mTORC1. Science 2013;340:1100-1106.

11. Yuskaitis CJ, Jones BM, Wolfson RL, et al., A mouse model of DEPDC5-related epilepsy: neuronal loss of Depdc5 causes dysplastic and ectopic neurons, increased mTOR signaling, and seizure susceptibility. Neurobiol Dis 2018;111:91-101.

12. Wang $Y, J i T$, Nelson $A D$, et al. Critical roles of $\alpha$ ll spectrin in brain development and epileptic encephalopathy. J Clin Invest 2018;128: 760-773.

13. Pollen AA, Nowakowski TJ, Chen J, et al. Molecular identity of human outer radial glia during cortical development. Cell 2015;163:55-67.

14. Korn MJ, Mandle QJ, Parent JM. Conditional disabled-1 deletion in mice alters hippocampal neurogenesis and reduces seizure threshold. Front Neurosci 2016;10:63.

15. Kane N, Acharya J, Benickzy S, et al. A revised glossary of terms most commonly used by clinical electroencephalographers and updated proposal for the report format of the EEG findings. Revision 2017. Clin Neurophysiol Pract 2017;2:170-185.

16. Watson BO, Levenstein D, Greene JP, et al. Network homeostasis and state dynamics of neocortical sleep. Neuron 2016;90:839-852.

17. Schwartz TH. Cortical dyplasia: complete resection correlates with outcome ... but, complete resection of what? Epilepsy Curr 2009;9:100-102.

18. Chen F, Rosiene J, Che A, et al. Tracking and transforming neocortical progenitors by CRISPR/Cas9 gene targeting and piggyBac transposase lineage labeling. Development 2015;142:3601-3611.

19. Cepeda C, André VM, Vinters HV, et al. Are cytomegalic neurons and balloon cells generators of epileptic activity in pediatric cortical dysplasia? Epilepsia 2005;46(suppl 5):82-88.

20. Calcagnotto ME, Paredes MF, Tihan T, et al. Dysfunction of synaptic inhibition in epilepsy associated with focal cortical dysplasia. J Neurosci 2005:25:9649-9657.

21. Hu JS, Vogt D, Sandberg M, Rubenstein JL. Cortical interneuron development: a tale of time and space. Development 2017;144: 3867-3878.

22. Carvill GL, Crompton DE, Regan BM, et al. Epileptic spasms are a feature of DEPDC5 mTORopathy. Neurol Genet 2015;1:e17. 